\title{
La palabra del agua en la narrativa de George Sand: Ce que dit le ruisseau
}

\author{
Lozano Sampedro, $M^{\mathrm{a}}$ Teresa
}

Universidad de Salamanca, tlozano@usal.es

\begin{abstract}
Resumen
El imaginario del agua en la narrativa sandiana reviste una gran riqueza. Baste recordar al respecto el amplio simbolismo del puente, el vado o la tormenta, motivos recurrentes en novelas tan conocidas como La Petite Fadette, La Mare au Diable o François le Champi. El propósito de nuestro estudio se centrará en el análisis de un relato muy breve, bastante olvidado por la crítica, titulado Ce que dit le ruisseau (1863). Este relato presenta claramente una idea esencial de la escritora - la necesidad de escuchar la voz de la Naturaleza - que constituirá el leitmotiv de su última obra: los trece Contes d'une grand'mère (publicados en volumen en 1873 y 1876). En Ce que dit le ruisseau, el lector asiste a un juego de ecos que plantea, en términos de interpretación y traducción, la relación entre la naturaleza y la inspiración artística. La dialéctica entre lo imaginario y lo real se presenta a través de dos voces discordantes, la de un poeta y la de un científico naturalista, a las que se unen la voz del agua y la de una enigmática ninfa. El viaje interiorizado y el sueño iniciático acaban por insertarse en la realidad en este relato polifónico y metafórico, como metafóricos son los Contes d'une grand'mère, a algunos de los cuales aludiremos. $Y$ también, como en ellos, el viaje y el sueño son inseparables de la primacía de la oralidad. El imaginario eminentemente terrestre de George Sand cede aquí la palabra al agua para afirmar la constante transformación y la eterna renovación de todo lo existente. En definitiva, Ce que dit le ruisseau es la expresión de un concepto múltiples veces expresado por la escritora: el de la incesante actividad de la Naturaleza, «reina de las hadas» que se opone a la existencia de la nada.
\end{abstract}

Palabras clave: George Sand; relato breve; polifonía; naturaleza; imaginario acuático.

\section{Résumé}

Dans la narrative sandienne, l'imaginaire de l'eau présente une grande richesse. Il suffit de se rappeler, à cet égard, certains motifs récurrents tels que le pont, le gué ou l'orage, dont la symbolique est si importante dans les célèbres romans La Petite Fadette, La Mare au Diable et François le Champi. Le propos de notre étude consistera dans l'analyse d'un récit très bref, souvent négligé par la critique, intitulé Ce que dit le ruisseau (1863). Ce récit expose clairement le besoin d'écouter la voix de la Nature, idée essentielle de l'écrivaine qui constituera le leitmotiv de sa dernière œuvre: les treize Contes d'une grand'mère (publiés en volume en 1873 et en 1876). Dans Ce que dit le ruisseau, le lecteur assiste à un jeu d'échos qui pose, en termes d'interprétation et de traduction, le problème de la relation entre la nature et l'inspiration de l'artiste. La dialectique entre l'imaginaire et le réel est configurée par deux voix discordantes, celle d'un poète et celle d'un savant naturaliste, auxquelles viennent s'ajouter la voix de l'eau et celle d'une nymphe très énigmatique. Comme dans les Contes d'une grand'mère, dont le sens est toujours métaphorique - nous en mentionnerons brièvement quelques-uns tout au long de notre étude - dans ce récit polyphonique et métaphorique le voyage intériorisé et le rêve initiatique finissent par s'insérer dans la réalité. Et aussi comme dans ces contes, le voyage et le rêve sont inséparables de l'oralité primordiale. L'imaginaire de George Sand, qui est essentiellement terrestre, cède ici la parole à l'eau pour affirmer la constante transformation et l'éternel renouvellement de tout ce qui existe. En définitive, Ce que dit le ruisseau constitue l'expression d'une idée souvent exprimée par l'écrivaine: l'activité incessante de la nature, «reine des fées» qui s'oppose à l'existence du néant.

Mots-clés: George Sand; récit bref; polyphonie; nature; imaginaire aquatique. 


\begin{abstract}
Imagination of water in George Sand's narrative is particularly rich. Suffice it to recall the broad symbolism of the bridge, the ford and the storm, all recurring motifs in well-known novels such as La Petite Fadette, La Mare au Diable or François le Champi. The purpose of our study is to analyse Ce que dit le ruisseau (1863), a quite forgotten very short story. The tale clearly presents a gist - the need of listening to Nature's voice- which will be the leitmotiv of her last work, the thirteen Contes d'une grand'mère (collected and published in 1873 and 1876). In Ce que dit le ruisseau, the reader witnesses an echoing game performing, in terms of interpretation and translation, the relationship between Nature and artistic inspiration. Two dissenting voices, a poet and a naturalistic scientist, join up the voices of both the water and an enigmatic nymph, to work the dialectic between reality and imagination. The inwards journey and the initiation dream penetrate reality in this polyphonic and metaphorical story, just as metaphorical as the Contes d'une grand'mère, that will partly be mentioned. And yet, like them, travelling and dream are inseparable from orality's primacy. Here, George Sand's imagination, mainly focused on Earth, gives the pool to the water in order to state the unbroken transformation and the eternal renewal of whatever exists. To sum up, Ce que dit le ruisseau puts one of the author's most repeated concepts across: namely, the endless activity of Nature, «The Queen of Fairies», as opposed to the existence of the void.
\end{abstract}

Keywords: George Sand; Short Story; Polyphony; Nature; Imagination of Water.

\title{
Introducción
}

Profunda conocedora de las ciencias naturales desde 1855, George Sand se apasiona igualmente por la zoología, la botánica y la mineralogía Y esta pasión imprime una huella permanente en su obra. Pero este afán naturalista resulta inseparable de su vertiente poética. La necesidad de escuchar la voz, la palabra de la naturaleza es un motivo recurrente en su narrativa. Prueba de ello es su última obra, los Contes d'une grand'mère, que ocupan un lugar importante dentro de su inmensa producción literaria. Estos trece cuentos de longitud diferente cuyas destinatarias directas son sus nietas Aurore y Gabrielle a quienes los contaba en las noches de Nohant antes de dormir, rebasando ampliamente el ámbito de la literatura infantil, nos ofrecen una profunda reflexión sobre el ser humano, el arte, la vida y la muerte. En ellos, la Palabra de la naturaleza, a veces explícitamente nombrada en ciertos títulos como Ce que disent les fleurs (Le Temps, 14 de julio de 1875) o Le Chêne parlant (Revue des Deux Mondes, 15 de octubre de 1875) ${ }^{1}$ se destaca como elemento clave. Y es que estos cuentos son su canto del cisne: «La dernière œuvre d'une femme qui a tant écrit est un hymne à la parole» (Didier, 2004: 53).

Al igual que en los Contes d'une grand'mère, la palabra, la metáfora y el viaje iniciático en sueños aparecen como elementos indisociables en un relato anterior, el que constituye el objeto de nuestro estudio: Ce que dit le ruisseau (1863). Poco conocido y estudiado, este relato que, a diferencia de los Contes d'une grand'mère, no se inscribe en el género de lo maravilloso, presenta sin embargo, como ellos, un leitmotiv de la obra sandiana: para descubrir las leyes de la naturaleza es preciso ver, observar, pero sobre todo saber oír, escuchar. La oralidad jalona la estructura de este brevísimo relato polifónico, en el que las diversas voces, a veces discordantes, expresan la aparente dicotomía entre la realidad y la imaginación a través de la oposición entre la ciencia y el arte. En el preámbulo de Laura, voyage dans le cristal $^{2}$, novela un año posterior al relato que será objeto de nuestro estudio, un personaje afirma:

L'artiste est né voyageur; tout est voyage pour son esprit, et, sans quitter le coin de son feu ou les ombrages de son jardin, il est autorisé à parcourir tous les chemins du monde. Donnez-lui n'importe quoi à lire ou à regarder, étude aride ou riante; il se passionnera pour tout ce qui lui sera nouveau. Il s'étonnera naïvement de n'avoir pas encore vécu

\footnotetext{
${ }^{1}$ Los Contes d'une grand'mère aparecieron en principio en las publicaciones periódicas Le Temps y la Revue des Deux Monde) antes de su recopilación en dos volúmenes, en 1873 y en 1876 .

${ }^{2}$ Publicada inicialmente en enero de 1864 en la Revue des Deux Mondes, el título fue evolucionando desde Géode pasando por Voyage dans le cristal, hasta la publicación en volumen en 1865 con su título definitivo.
} 
dans ce sens-là, et il traduira le plaisir de sa découverte sous n’importe quelle forme, sans avoir cessé d'être lui-même (Sand, 1980a: 31-32).

Bajo el signo de esta traducción y bajo el paradigma privilegiado en la obra sandiana del «artista viajero», personaje tan recurrente en la obra de Hoffmann, se sitúa Ce que dit le ruisseau, en el que asistimos, mediante un juego de ecos, a la relación entre la voz de la naturaleza y la poesía. Y como en todo relato iniciático y de aprendizaje, en este breve texto «la romancière cherche à préserver les prestiges de l'imaginaire sans nuire à une traduction réaliste de l’homme et du monde» (Schaeffer, 1981: 75).

\section{El desafío al poeta o los dos viajes}

Ce que dit le ruisseau narra en primera persona la experiencia de un poeta, Théodore, que realiza con su amigo Lothario, científico naturalista, un viaje hasta el río la Creuse, en los alrededores de Nohant y de Gargilesse. Desde el inicio, la ensoñación poética viene a definir la personalidad de Théodore: «J’étais fatigué quand je m’arrêtai au bord du ruisseau babillard. La nymphe qui est de ma connaissance, vu que je la rencontre souvent dans la forêt et dans la montagne, vint à moi toute courroucée» (Sand, 1981: 227). Esta extraña ninfa, «allégorique gardienne du ruisseau, vient d’abord s'interposer entre le ruisseau el le promeneur, accusant ce dernier de mentir, [...] c'est-à-dire de n'être pas assez poète pour comprendre ce que dit le ruisseau» (Berger, 1988: 97). La visión se burla de la incapacidad del poeta de comprender y hablar el lenguaje de la naturaleza y por lo tanto, de traducirlo: «Vous autres rêveurs, vous êtes d'une insupportable curiosité. Vous vous piquez de deviner nos secrets, et vous les traduisez à tort et à travers. Va-t-en ! tu n'as que faire dans mon jardin et dans ma prairie (Sand, 1981: 227)». Ante las súplicas de Théodore de dejarle descansar al borde del arroyo, la ninfa acabará cediendo a su ruego, pero no sin proferir ciertas amenazas que acabará cumpliendo. Y toda una poética de la fraîcheur des eaux printanières se despliega ante el lector en este relato en el que el agua acabará revelándose como «la maîtresse du langage fluide, du langage sans heurt, du langage continu, continué» (Bachelard, 1942: 250). Théodore, seguro de que el arroyo habla, intenta comprender sus palabras: «Il tombait, tombait ; il courait, courait, mais surtout, et il me sembla que c’était là son affaire de prédilection, il parlait, parlait; il ne déparlait pas » (Sand, 1981: 228). Y el texto empieza así a insertarse en el dominio de l’imagination parlante: «Comment ne pas vivre [...] le parler liquide, le parler gouailleur, l’argot du ruisseau !» (Bachelard, 1942: 253). Pero la ensoñación del narrador se verá interrumpida bruscamente por la voz de la ciencia:

- Bah ! Me dit Lothario, qui était venu me rejoindre et qui me surprit aux écoutes, il résonne, il gazouille, il murmure, comme disent les romances; mais il ne parle pas, va ! Tu peux donner carrière à ton imagination ; mais moi, je te jure qu'il ne dit rien du tout (Sand, 1981: 228).

En la obra de George Sand, mujer que tanto viajó, el viaje «ne prend tout son sens que s’il s’intériorise, à partir de n’importe quelle réalité» (Schaeffer, 1981: 22). El paseo de Théodore al borde del río llegará a ser una fuente de exploración del universo porque, como es frecuente en la narrativa sandiana, el viaje interior configura la estructura del relato:

Ce petit voyage a en effet été l'occasion d'une épreuve transformatrice que sa structure et ses motifs apparentent à une expérience religieuse : le récit retrace les tribulations d’un croyant en la poésie dont la foi est ébranlée puis reconquise à la faveur d’une promenade qui revêt, en ce sens, un caractère initiatique (Berger, 1988: 96).

En efecto, la ruta del poeta se ve ya desde el inicio dividida entre «deux voies dont la divergence va prendre la forme d'un conflit de voix» (Berger, 1988: 96). Divergencia que, de manera paradójica, deriva en cierta confluencia. El diálogo del poeta con «deux figures antagonistes qui représentent les termes symétriquement opposés de son débat» (Berger, 1988: 96-97) se estanca en una misma conclusión: tanto la ninfa, «trompeuse vision prise au répertoire des conventions 
poétiques» (Berger, 1988: 97), como Lothario con su escepticismo de científico racionalista, acusan a Théodore de no comprender lo que dice el arroyo: «Théodore ment, ou plus exactement, il "traduit”, comme le disent la nymphe et Lothario, parce qu'il n’est pas assez poète ou parce qu’il l'est trop» (Berger, 1988: 97). Sin embargo, Théodore afirma: «L'étude des choses réelles était aussi le but de ma promenade» (Sand, 1981: 228). Y por ello nos parece encarnar el concepto sandiano de la unión indisoluble entre la voz de la naturaleza y la realidad cotidiana: «Le merveilleux est de tous les jours, mais il faut savoir le voir. Le féerique ne consiste pas en une libération des lois de la nature, mais au contraire dans la découverte de ces lois» (Didier, 2004: 52). Todo tiene un mensaje porque, como finalmente la palabra del arroyo revelará, todo lo existente en la creación forma parte del gran todo universal. Y este mensaje no es gratuito, sólo espera a ser escuchado. Al contrario que Lothario, Théodore niega el azar:

- Je te jure [...] que ce ruisseau ne chante pas au hasard. Nous sommes des sourds qui voulons faire les esprits forts, et nous parlons des voix de la nature comme les aveugles des couleurs. Si nous avions un peu d'intelligence et beaucoup de patience, nous finirions par comprendre ce que dit ce filet d'eau (Sand, 1981: 228) ${ }^{3}$.

El poeta invita a su amigo a escuchar el canto del ruiseñor, el murmullo de las abejas, a admitir la existencia del lenguaje de las hormigas, de los insectos minúsculos cuyos sonidos son imperceptibles... Si en uno de los Contes d'une grand'mère, Le Marteau Rouge (Le Temps, 28 de julio de 1875) la narradora afirma: «Tout dans la nature a une voix, mais nous ne pouvons attribuer la parole qu'aux êtres» (Sand, 1979a: 203), en Ce que dit le ruisseau el debate sobre los límites de lo animado y lo inanimado acabará por conferir su sentido al texto entero. La argumentación de Théodore sobre la palabra del agua es rebatida así por Lothario: «Si tu confonds maintenant le langage des animaux avec celui des choses, tu confonds les êtres avec la matière inanimée, et il n’y a plus moyen de causer avec un fou» (Sand, 1981: 230).

El título Ce que dit le ruisseau presenta una evidente analogía con el de uno de los Contes d'une grand'mère, anteriormente mencionado: Ce que disent les fleurs. Y es precisamente el lenguaje de las flores el último punto de la discusión entre el poeta y el científico. El lector asiste a todo un juego de preguntas y respuestas que puede resumirse desde la perspectiva de la aceptación o el rechazo de la metáfora, tal y como se desprende del ejemplo puesto por Lothario a Théodore:

Si tu me disais: “Je vais écouter le ramage des fleurs”, je te répondrais que je te sais capable de tout; mais je ne verrais là que l'exagération poétique d’une déduction assez fondée, tandis que, devant ta prétention de surprendre le langage d'un ruisseau, je te salue comme le plus grand fou que la manie littéraire ait jamais produit (Sand, 1981: 230-231).

En este relato, George Sand confiere a la imaginación poética lo que en los Contes d'une grand'mère presentará como una facultad de la infancia, que los adultos ya han perdido. La voz de Lothario - voz del científico puro - y la del poeta Théodore podrían corresponderse respectivamente con las voces del profesor de botánica y de la niña protagonista del cuento Ce que disent les fleurs:

Quand j'étais enfant, [...] j'étais très-tourmentée de ne pouvoir saisir ce que les fleurs se disaient entre elles. Mon professeur de botanique m'assurait qu'elles ne disaient rien ; soit qu'il fût sourd, soit qu'il ne voulût pas me dire la vérité, il jurait qu'elles ne disaient rien du tout.

Je savais bien le contraire. Je les entendais babiller confusément, surtout à la rosée du soir ; mais elles parlaient trop bas pour que je pusse distinguer leurs paroles (Sand, 1979b: 183).

\footnotetext{
${ }^{3}$ La inteligencia y la paciencia son elementos clave en los Contes d'une grand'mère. Frecuentemente, las heroínas, niñas o adolescentes, cuentan con la ayuda de un hada, de una mujer-guía cuya única función es precisamente la del hada: decir, orientar por medio de la palabra. Pero serán ellas mismas quienes deberán, con su esfuerzo continuado, captar la voz de la naturaleza que les ayude a descubrir su vocación. Le Nuage rose (Revue des Deux Mondes, 1 de agosto de 1872) y Le Château de Pictordu (Le Temps, del 5 al 23 de marzo de 1873) son claros ejemplos de ello.
} 
Una noche, escondida en el jardín, la niña acabará por escuchar en sueños la voz de las flores. Y también es un sueño el que revelará a Théodore las palabras del arroyo. Revelación en solitario, como lo es toda revelación iniciática: «Lothario ne voulut pas m’écouter davantage. Une libellule qui passait lui parut pour le moment plus intéressante que ma conversation, et il s'éloigna à sa poursuite» (Sand, 1981: 231).

En definitiva, por su afán de clasificar lo existente en palabras, en definiciones, por su empeño en establecer la diferencia tajante entre los seres y las cosas, «Lothario résiste à l’attraction de la métaphore, à sa faculté de transporter celui qui se laisse prendre au charme rusé du langage» (Berger, 1988: 100-101). Carente de imaginación, se verá así privado del viaje revelador, privilegio exclusivo del poeta. Viaje que, como veremos, tiene su origen en la primacía de la oralidad.

\section{La palabra interior o el principio de la revelación}

Como todo relato de iniciación, Ce que dit le ruisseau es un relato de búsqueda de la identidad. El poeta, solo ante el arroyo, se ve confrontado a sus propias dudas. Y la otra voz discordante irrumpe de nuevo, la voz de la ninfa burlona que parece estar de acuerdo con Lothario:

- Cherche, va ! disait-elle, cherche ce qui se dit dans l'eau, dans le vent, dans le sable ou dans la nuée ! Ton ami l'a trouvé, lui ; il ne se dit rien du tout ! Les êtres seuls sont doués de la parole, et moi, je ne suis rien, je suis muette, muette [...] comme ma roche et comme mon ruisseau ! (Sand, 1981: 231).

Pero la tentación de creer en el mutismo de la naturaleza es pronto superada por Théodore pues, en el pensamiento de Sand, «pour l'œil attentif, pour le cœur réceptif, les merveilles se prodiguent dans chaque geste, dans chaque instant qu’il nous est accordé de vivre» (Anoll, 2004 : 194). Sueño o alucinación, la ninfa, que ahora es invisible, hará del poeta la víctima de un encantamiento que supone un reto, una disyuntiva. Théodore se verá privado de la movilidad - «je reconnus avec stupeur que j’étais enchaîné là par une force magique» (Sand, 1981: 232) - y ésta será la condición impuesta para su liberación: «- Alors, renonce à la poésie et jure que tu ne chercheras plus rien en dehors de la science» (Sand, 1981: 232). Renuncia imposible para Théodore, puesto que la búsqueda de la palabra del arroyo no es, en definitiva, sino la búsqueda del Arte: «La notion poétique qui ne vous surprend comme une impérieuse révélation n’est pas la poésie, [...] mais je pensais que cette révélation devait être écoutée comme une voix de la nature elle-même» (Sand, 1981: 231).

En el conjunto de los Contes d'une grand'mère, es el más largo de todos ellos, Le Château de Pictordu, el más representativo de la relación entre la naturaleza y el arte en el pensamiento de Sand. La dialéctica entre el sueño y la realidad, siempre presente, es inseparable del poder de la palabra en este relato en que una estatua habla a una niña, Diane, que busca su identidad como dibujante y que acabará siendo también una gran pintora. Lo que nos interesa destacar aquí, en relación con el tema que nos ocupa ${ }^{4}$, es este concepto de revelación de la naturaleza en relación con la obra artística. Al título del primer capítulo del cuento («La statue parlante»), responderá en eco el título del último, «Discours de la statue», en el cual, tras un largo proceso iniciático y de aprendizaje, Diane, ya adulta y gran dibujante, volverá de visita al castillo de su primera iniciación. Y un paseo nocturno por los alrededores despierta en ella la necesidad «de résumer sa courte vie au milieu de cette nature qui semblait absorbée dans la méditation de l'éternité» (Sand, 1979c: 105). Admirando «les beaux effets du clair de lune dans les ruines» (Sand, 1979c: 105), sus dudas se verán disipadas por la voz de la estatua que le señala «la route de l'idéal» (Sand, 1979c: 107). Esta voz, que no es evidentemente sino la propia voz interior de la artista, constituye una «révélation intérieure» (Sand, 1979c: 107) que desencadenará la revelación exterior. A la mañana siguiente, Diane siente el impulso de contemplar el paisaje a la luz del día, y quedará admirada y sorprendida ante el cuadro vivo creado por la Naturaleza. Percibirá «la vie magique de la lumière», sentirá «pour la première fois, [...] l’ivresse de la couleur» y hará el descubrimiento esencial que la iniciará en el arte de la pintura gracias a «cette révélation qui lui venait du ciel et de la terre, du feuillage et des eaux, des herbes et du rocher» (Sand, 1979c: 110).

En Ce que dit le ruisseau, es también la palabra interior el germen de la revelación. El sortilegio de la ninfa es doble, pues a la inmovilidad de Théodore se añade el mutismo: «Je te retire aussi la parole, car je ne veux pas que tu ennuies

\footnotetext{
${ }^{4}$ No entraremos en detalles sobre la complejidad y el interés de este cuento, ya que ello rebasaría los límites de nuestro estudio.
} 
mon ruisseau par de sottes questions» (Sand, 1981: 232). Aparente crueldad que no es tal, puesto que lo que la ninfa propone es en realidad la iniciación en el sueño iniciático: «Dès que la vraie parole se formulera dans ton esprit, tu n’auras pas besoin de m’en faire part. La vérité te délivrera toute seule et sans mon aide, puisqu'elle sera en toi» (Sand, 1981: 232). El texto va adentrándose en el terreno de la mímesis a través de la oralidad. El poeta, mudo e inmóvil, antes de comprender las palabras de arroyo «sent tout à coup affluer en lui une parole intérieure, non bruyante, toute oreille - une écriture ? -, dont le débit devient torrentiel ; elle s’adresse (ou elle adresse celui qu'elle submerge) au ruisseau» (Berger, 1988: 99). Y es que George Sand dota a su personaje, que mucho se asemeja a ella misma, de la cualidad que Bachelard definiría como «une oreille poétisée», capaz de unificar «des voix discordantes quand elle se soumet au chant de l’eau comme à un son fondamental» (1942: 260). Para escuchar la palabra del agua, hay que hablar al agua - «Il faudra que l'être malheureux parle à la rivière» (1942: 261) - y es el discurso humano el que arrancará sus palabras al arroyo, palabras que vendrán a corroborar las convicciones del poeta y que, efectivamente, le liberarán, tal y como la ninfa había prometido. Discurso que, más que como una meditación, se presenta como una revelación de la voz de la naturaleza, expresando una de las ideas clave del pensamiento sandiano:

Tout parle et tout chante sous le ciel et probablement dans le ciel : qui osera décider que, dans la nature, il y ait une voix inutile, un chant qui n'exprime rien ? Non, il n’y a pas même un cri, un souffle, un rugissement, un murmure, une explosion, un bruit enfin qui ne signale ou ne traduise une action, un mode d'existence ou un accident logiquement survenu dans le cours de la vie universelle (Sand, 1981: 233).

Todo habla y canta porque todo traduce el eterno fluir del universo. Y es, por ello, misión del artista traducir a su vez estas voces pues, como dice Théodore, «où j'entends un langage, j’ai la certitude qu’il y a l'expression d'une émotion ou d'une sensation, ou tout au moins d'une vitalité quelconque» (Sand, 1981: 233). En Le Chien et La Fleur sacrée, otro de los Contes d'une grand'mère (Revue des Deux Mondes, 1 de noviembre de 1875) un personaje llamado M. Lechien realiza esta afirmación: «Toute chose est un élément de transformation. La plus grossière a encore sa vitalité latente dont les sourdes pulsations appellent la lumière et le mouvement : l’homme désire, l’animal et la plante aspirent, le minéral attend» (Sand, 1979d: 72) ${ }^{5}$. Será este el mensaje del agua que Théodore intuye antes de escucharlo: todo lo existente está sometido al «incoercible mouvement», debido a «l'éternelle mutation progressive des êtres et des germes qui les contiennent, germes répandus partout et que nous appelons des choses, faute d’un nom qui caractérise leurs fonctions multiples et indiscernables» (Sand, 1981: 235). El arroyo es « un organe de la création » que forma parte de «ce grand être qu’on appelle la terre» (Sand, 1981: 233). Por ello, «Théodore et le ruisseau se font écho jusqu’à n’être plus que l'écho l'un de l'autre, et même l'écho de l'écho» (Berger, 1988: 103). En la continua cadena universal de transformaciones, el arroyo ha sido nube y ha traducido el canto de los pájaros que a su vez traducirá el poeta, pues «tout est écho dans l'univers» (Bachelard, 1942: 259).

\section{La respuesta del arroyo: la eterna renovación}

Otro de los Contes d'une grand'mère, La Fée Poussière (Le Temps, 11 de agosto de 1875) constituye «un récit porteur d’une création mythique» (Veys, 2003: 60) que terminará por una revelación palingenésica. En él, el hada Tierra desvela a una niña el secreto de la creación en estos términos:

Je sème la destruction pour faire pousser le germe. Il en est ainsi de toutes les poussières, qu'elles aient été plantes, animaux ou personnes. Elles sont la mort après avoir été la vie, et cela n’a rien de triste, puisqu'elles recommencent toujours, grâce à moi, à être la vie après avoir été la mort (Sand, 1979e: 244).

\footnotetext{
${ }^{5}$ Este cuento trata el tema de la metempsicosis. La adhesión de George Sand a esta doctrina, especialmente desde su amistad con Pierre Leroux, viene determinada sobre todo por su creencia en la eterna renovación universal.
} 
Es este el sentido de la palabra interior del poeta Théodore, dirigida al arroyo: «Ruisseau qui fus nuage, tu nous diras tout ce que tu as fécondé dans ta vie errante ? [...] Pluie secourable, combien de moissons n’as-tu pas sauvées, combien de fleurs charmantes et suaves n’as-tu pas fait revivre !» (Sand, 1981: 235). Y planteará al agua su mayor duda:

O nuage béni ! si petit que tu fus, tu as fait de grandes choses, et la parole te serait refusée pour les dire !

Mais quoi ! n’est-tu plus qu'un mince filet d'eau enchaîné à cette roche, contenu dans l'urne de cette naïade et condamné à faire pousser un tapis de jacinthes ou à développer la hampe des hautes primevères ? (Sand, 1981: 236).

El título del relato, Ce que dit le ruisseau, es significativo, pues en realidad el discurso del arroyo es muy breve. La palabra del agua no es más que la confirmación de la palabra interior del poeta, y esta palabra se resume en el concepto del eterno renacer:

Non pas, non pas ! répondit le ruisseau, je suis ici et je suis ailleurs. Je féconde ce qui vit sous tes pieds, et je suis fécondé moi-même à toute heure en remontant dans le libre domaine de l'air. Mon évaporation est comme une sueur de vie qui se répand sur tout ce qu'elle touche et qui se reforme en nuage pour courir encore sur la cime des grands chênes (Sand, 1981: 236).

En otros términos, el arroyo, que se define como «une des manifestations particulières du grand fluide vital [...] qui accompagne et revêt la terre dans son voyage à travers l'infini» (Sand, 1981: 236), arrastra al poeta (y al lector) a un vertiginoso y magnífico viaje:

\begin{abstract}
Je ne puis dire où je vais et où je ne vais pas [...]; mais Dieu les connaît, mes beaux voyages, et toute la nature en profite ; et moi, je m'en réjouis sans cesse, et toujours je ris, je cours, je chante, je raconte, je confie, je révèle, je bois et donne à boire, je sème et je récolte, je prends et je donne ; tout me nourrit, même ton haleine, et je nourris tout, même ta pensée ! (Sand, 1981: 236).
\end{abstract}

El agua y el ser humano se nutren mutuamente, porque Théodore ha sabido buscar en la naturaleza la palabra poética «qui consisterait simplement à faire écho au chant de la terre» (Berger, 1988: 104) y que por ello permite al hombre «de rester lié ou de faire retour au tout dont il émane» (Berger, 1988 : 104). La misteriosa ninfa tenía razón en sus afirmaciones. Théodore se ha liberado del encantamiento al descubrir la verdadera palabra dentro de sí mismo:

Et le ruisseau, dont j'avais traduit le langage, me fit connaître que je ne l'avais pas fait mentir, car j'entendis qu'il disait distinctement, comme un résumé de mes hypothèses: Toujours, toujours partout, dans tout, pour tout, toujours!

Et il recommençait sans se lasser, car c'est tout ce qu'il pouvait dire, et il ne pouvait rien dire de plus beau (Sand, 1981: 236).

Este relato de una breve excursión a la naturaleza sigue una estructura circular. El reencuentro final con Lothario, que marca la vuelta de Théodore a la realidad, supone nuevamente el reencuentro de las dos voces discordantes. Y como el poeta dice, «s'il n'est pas toujours possible à l'homme de comprendre l'hymne de la nature, il lui est toujours permis de le deviner» (Sand, 1981: 237). Debate entre Lothario y Théodore que queda, como al principio, sin resolver:

- Poésie, dit-il en levant les épaules, horreur du vrai !

- Non pas, répondis-je, culte du vrai, mais traduction libre! (Sand, 1981: 237). 
En el citado preámbulo de la novela Laura, voyage dans le cristal, encontramos esta afirmación:

Pas plus que les autres humains, l'artiste ne choisit son genre de vie et la nature de ses impressions. Il reçoit du dehors le soleil et la pluie, l'ombre et la lumière, comme tout le monde. Ne lui demandez pas de créer en dehors de ce qui le frappe. Il subit l'action du milieu qu'il traverse, et c'est fort bien fait, car il s'éteindrait et deviendrait stérile le jour où cette action viendrait à cesser (Sand, 1980a: 32).

Ce que dit le ruisseau nos parece constituir una interesante reflexión sobre la relación entre la naturaleza y el arte cuyo sentido final viene a corroborar lo que George Sand expresa en sus Légendes rustiques (1858): «Tout être et toute chose protestent contre le néant» (Sand, 1980b: 6).

\section{Conclusión}

En la carta que encabeza Un hiver à Majorque (1842) - la Lettre d'un ex-voyageur à un ami sédentaire - George Sand afirma: «Mes plus beaux, mes plus doux voyages, je les ai faits au coin de mon feu, les pieds dans la cendre chaude et les coudes appuyés sur les bras râpés du fauteuil de ma grand'mère» (Sand, 1997: 11). Texto que nos parece especialmente elocuente de la importancia del viaje inmóvil, tal y como el que realiza el poeta Théodore.

¿Quién habla y quien escucha en Ce que dit le ruisseau? «L'art a besoin de s’instruire sur des reflets, la musique a besoin de s'instruire sur des échos. C'est en imitant qu’on invente. On croit suivre le réel et on le traduit humainement», dice Bachelard (1942: 259). El lector dotado de la capacidad de escuchar, pues en éste como en otros relatos de George Sand en el origen está la palabra, se siente tentado de compadecer a Lothario, incapaz de sentir esta bella formulación bachelardiana: «Le ruisseau vous apprendra à parler [...] malgré les peines et les souvenirs, il vous apprendra [...] l'énergie par le poème. Il vous redira, à chaque instant, quelque beau mot tout rond qui roule sur des pierres» (1942: 262).

\section{Referencias bibliográficas}

ANOLL, Lídia (2004). «Contes d’une grand'mère: un autre visage de George Sand ?», en George Sand 1804-2004. L'île et la dame de Nohant. Estudi General LUL.LIA de Mallorca, p. 191-208.

BACHELARD, Gaston (1942). L'eau et les rêves (Essai sur l'imagination de la matière). Paris: José Corti.

BERGER, Anne (1988). «Ce que dit le ruisseau», en Études françaises, vol. 24, n 1, p. 95-105. <http://id.erudit.org/iderudit/035744ar>. DOI: 10.7202/035744ar. [Consulta: 12 de febrero de 2016]

DiDIER, Béatrice (2004). George Sand. Paris: ADPF (Association pour la diffusion de la pensée française).

SAND, George (1979a). Le Marteau Rouge (Contes d'une grand'mère, vol. 2): Éditions d'aujourd'hui, Imprimerie de Provence.

SAND, George (1979b). Ce que disent les fleurs (Contes d'une grand'mère, vol. 2): Éditions d'aujourd'hui, Imprimerie de Provence.

SAND, George (1979c). Le Château de Pictordu (Contes d'une grand'mère (vol. 1): Éditions d'aujourd'hui, Imprimerie de Provence.

SAND, George (1979d). Le Chien et La Fleur sacrée (Contes d'une grand'mère, vol. 2): Éditions d'aujourd'hui, Imprimerie de Provence.

SAND, George (1979 e). La Fée Poussière (Contes d'une grand'mère, vol. 2): Éditions d'aujourd'hui, Imprimerie de Provence.

SAND, George (1980a). Laura, Voyage dans le cristal. Paris: Union Générale d’Éditions.

SAND, George (1980b). Légendes rustiques. Paris: Éditions Libres-Halliers.

SAND, George (1981). Ce que dit le ruisseau. Paris: Union Générale d’Éditions.

SAND, George (1997). Un hiver à Majorque. Mallorca: Ingrama Editorial.

SCHAEFFER, Gérald (1981). Espace et temps chez George Sand. Neuchâtel (Suisse): La Baconnière.

VEYS, Sylvie-Victoire (2003). «George Sand et l'imagination matérielle selon Gaston Bachelard», en Les Amis de George Sand (Nouvelle Série), $n^{\circ}$ 25, p. 55-66. 